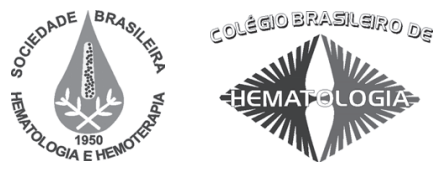

\title{
Osteonecrose dos maxilares associada ao uso de bisfosfonatos: importante complicação do tratamento oncológico
}

\section{Bisphosphonate-associated jaws osteonecrosis: an important complication of oncology treatment}

Marco Antonio T. Martins ${ }^{l}$

Auro del Giglio ${ }^{2}$

Manoela D. Martins ${ }^{3}$

Vanessa C. S. Pavesi ${ }^{4}$

César A. Lascala

\begin{abstract}
Os bisfosfonatos são um grupo de medicamentos utilizados no tratamento de doenças malignas metastáticas e em outras doenças ósseas como osteoporose e doença de Paget. A despeito dos seus benefícios, uma importante complicação denominada de osteonecrose dos maxilares vem sendo observada nos pacientes usuários crônicos dos bisfosfonatos que se caracteriza clinicamente por exposições ósseas na região maxilofacial persistente, acompanhadas de osteomielite, geralmente sintomáticas e cujo tratamento é complexo. Este estudo tem por objetivo revisar a literatura sobre a osteonecrose associada ao uso dos bisfosfonatos, em especial, em oncologia, no período de 2003 a 2008. Serão apresentados e discutidos os fatores de risco, aspectos etiopatogênicos, clínicos, imagenológicos, terapêuticos e preventivos desta doença. Devido à dificuldade de tratamento da osteonecrose associada aos bisfosfonatos, o foco deve ser a prevenção, sendo o ideal a eliminação de quadros infecciosos orais antes da terapia com os bisfosfonatos ter sido iniciada e minimizar traumas em boca após o uso destes medicamentos. Rev. Bras. Hematol. Hemoter. 2009;31(1):41-46.
\end{abstract}

Palavras-chave: Revisão; fisiopatologia; osteonecrose; diagnóstico; terapia; manifestações bucais.

\section{Introdução}

Os pacientes com câncer e portadores de doenças metastáticas ósseas frequentemente exibem complicações que incluem dor, fratura patológica, compressão da medula espinhal e hipercalcemia, que causam piora da qualidade de vida, grande morbidade e mortalidade. ${ }^{1}$ Essas alterações são normalmente consequência do processo de metástase, que é resultado da ativação de osteoclastos, mediado por diferentes citocinas produzidas pelas células tumorais, o que ocasiona a reabsorção óssea permitindo o crescimento tumoral. ${ }^{2}$
Com o intuito de controlar essas complicações, nos últimos anos, os oncologistas vêm utilizando medicamentos denominados bisfosfonatos (BFs), que são análogos dos pirofosfatos, não metabolizados, capazes de se depositarem no osso e inibir a função osteoclástica. Esses medicamentos fazem parte do protocolo de tratamento para pacientes com moderada a severa hipercalcemia associada com câncer; pacientes com lesões osteolíticas associadas ao câncer de mama e mieloma múltiplo em conjunto com quimioterapia antineoplásica e para lesões osteolíticas originárias de qualquer tumor sólido. ${ }^{3,4}$ Isso resultou no uso

${ }^{1}$ Cirurgião-dentista. Professor de Curso de Odontologia da Uninove e Unimes.

${ }^{2}$ Médico oncologista. Coordenador do Programa de Oncologia do Hospital Israelita Albert Einstein e Professor Titular de Hematologia e

Oncologia da Faculdade de Medicina do ABC.

${ }^{3}$ Cirurgiã-dentista. Professora do Mestrado em Ciências da Reabilitação da Uninove.

${ }^{4}$ Cirurgiã-dentista.

${ }^{5}$ Professor Livre-docente da Disciplina de Radiologia da Faculdade de Odontologia da USP.

Universidade Nove de Julho (Uninove) - São Paulo-SP.

Correspondência: Marco Antonio Trevizani Martins

Rua Demóstenes, 636 apto 11 - Campo belo

04614-013 - São Paulo-SP - Brasil

Fonelfax: (+55 11) 32564421

Email: kekomartins@yahoo.com.br-manomartins@gmail.com 
rampante dos bisfosfonatos na maior parte das clínicas e hospitais de oncologia médica no mundo. Inúmeros trabalhos clínicos têm evidenciado a eficácia dos BFs na diminuição da lise óssea mediada por osteoclastos, redução da dor óssea e complicações esqueléticas. ${ }^{1,5-9}$

Existem vários tipos de BFs aprovados para uso clínico, divididos em gerações, sendo que as propriedades antireabsortivas dos BFs aumentam, aproximadamente, dez vezes entre as gerações da droga. Os denominados de endovenosos são os utilizados em pacientes oncológicos e os de uso oral para tratamento de outras doenças que ocasionam lise óssea, dentre elas a osteoporose. Os endovenosos incluem o pamidronato (Aredia $\left.{ }^{\circledR}\right)$, um BF de segunda geração e o ácido zoledrônico (Zometa $\left.{ }^{\circledR}\right)$, o mais potente BF de uso clínico, de última geração. Em comparação com o pamidronato, o ácido zoledrônico é significantemente mais efetivo no controle da hipercalcemia maligna e na redução do número de eventos relacionados às complicações ósseas. $\mathrm{O}$ ácido zoledrônico é administrado através de uma dose mensal de 4 $\mathrm{mg} / \mathrm{mês}$ durante um período de 15 minutos. Se bem tolerado, não é incomum que os pacientes mantenham esta terapia indefinidamente. ${ }^{4}$

Os BFs afetam o remodelamento ósseo em vários níveis, apesar dos mecanismos exatos ainda não terem sido completamente elucidados. ${ }^{10}$ No nível tecidual, os BFs inibem a reabsorção óssea, diminuindo o remodelamento ósseo, podendo alterar o grau de formação óssea. No nível celular, os BFs alteram os osteoclastos inibindo sua função de várias maneiras, tais como: inibição do recrutamento ${ }^{11} \mathrm{e}$ diminuição do tempo de vida dos osteoclastos ${ }^{12}$ e inibição da atividade osteoclástica na superfície óssea. ${ }^{13}$ No nível molecular tem se evidenciado que os BFs modulam as funções dos osteoclastos pela interação com receptores da superfície celular ou por enzima intracelular. ${ }^{14}$

Apesar de todos os benefícios da terapia com bisfosfonatos, essas drogas vêm sendo associadas, desde 2003, a uma debilitante complicação que afeta exclusivamente a mandíbula e a maxila denominada de osteonecrose dos maxilares. ${ }^{15-20}$

No presente estudo serão revisados os aspectos epidemiológicos, etiopatogênicos, clínicos, imagenológicos, condutas terapêuticas e preventivas da osteonecrose associada ao uso dos bisfosfonatos (ONMAB).

\section{Epidemiologia}

Dados epidemiológicos como prevalência não podem ser avaliados até o momento; entretanto, os trabalhos têm mostrado que, na terapia com bisfosfonatos endovenosos em pacientes com mieloma múltiplo, estima-se que $1,8 \%$ a $12,8 \%$ dos pacientes desenvolvam ONMAB. ${ }^{18}$

De acordo com Woo et al., ${ }^{21} 94 \%$ dos casos publicados de ONMAB são de pacientes fazendo uso de bisfosfonatos endovenosos devido a mieloma múltiplo ou carcino- mas metastáticos. Tendo em vista o aumento do conhecimento sobre esta condição, maior tempo de exposição aos BFs e maior acompanhamento dos pacientes, espera-se que a incidência de ONMAB deva aumentar. ${ }^{22}$

Durie et al. ${ }^{23}$ conduziram um estudo com auxílio da internet onde participaram 904 pacientes com mieloma, sendo que, destes, 62 tinham o diagnóstico de ONMAB e 54 tinham a suspeita de ter a doença. Destes pacientes, $71 \%$ tinham recebido tratamento com ácido zoledrônico e $29 \%$ com pamidronato. Neste estudo, após 36 meses de tratamento, $10 \%$ dos pacientes que receberam ácido zoledrônico e $4 \%$ dos tratados com pamidronato tiveram ONMAB. O tempo para o aparecimento da ONMAB foi, em média, de 18 meses para o ácido zoledrônico e 6 anos para o pamidronato.

A incidência de ONMAB em pacientes portadores de osteoporose, doença de Paget, osteogênese imperfeita, artrite reumatóide é bastante rara. Dados fornecidos pelo fabricante do alendronato (Merck), a incidência de ONMAB foi calculada em 0.7/100.000 pessoas/ano de exposição. ${ }^{24}$ Hesse et $a l .{ }^{25}$ realizaram uma revisão sistemática para identificar ONMAB em pacientes que utilizaram bisfofosfonatos por outra indicação que não no tratamento oncológico. Apenas 99 casos foram identificados, sendo que, destes, 85 pacientes tinham osteoporose, dez doença de Paget e dois artrite reumatóide.

\section{Aspectos clínicos}

De acordo com a Associação Americana de Cirurgiões Orais e Maxilofaciais, ${ }^{24}$ a osteonecrose dos maxilares associada ao uso de bisfosfonatos (ONMAB) se caracteriza clinicamente por exposições ósseas na região maxilofacial persistentes por mais de oito semanas, com história médica de uso de bisfosfonatos e sem história de radioterapia nos maxilares. A ONMAB pode ficar assintomática por várias semanas, meses ou anos. Alguns sinais e sintomas podem ser identificados antes do desenvolvimento clínico da ON tais como: dor, mobilidade dental, aumento de volume da mucosa, eritema, ulceração, drenagem de secreção em boca, exposição óssea, osteomielite e fratura patológica.

Na maior parte dos casos, as lesões se mostram sintomáticas quando há infecção e resposta inflamatória tecidual local. Apesar de estas complicações serem observadas tanto na maxila como na mandíbula, a maior incidência é na mandíbula e, frequentemente, ocorrem após extração dental, mas podem também ocorrer espontaneamente. ${ }^{16-20,26-28}$

Ruggiero et al. ${ }^{15}$ propuseram uma classificação clínica da ONMAB em três estágios: Estágio 1 se caracteriza pela exposição e necrose óssea assintomática; Estágio 2, por exposição e necrose óssea associada a dor e infecção; Estágio 3 , mostrando tecido ósseo necrótico e exposto em pacientes com dor, infecção, fratura patológica, fístula extraoral e extensa osteólise. 


\section{Patogênese}

A patogênese da ONMAB não está completamente elucidada, mas parece estar relacionada com as alterações vasculares e da estrutura óssea causadas pelos bisfosfonatos. Acredita-se que a ONMAB resulte de uma interligação entre metabolismo ósseo alterado pelos bisfosfonatos, trauma local, aumento da necessidade de reparo ósseo, infecção e hipovascularização. ${ }^{15,17,18}$ Assim, a necrose óssea seria o resultado da incapacidade do tecido ósseo afetado em reparar e se remodelar frente a quadros inflamatórios desencadeados por estresse mecânico (mastigação), exodontias, irritações por próteses ou infecção dental e periodontal. Adicionalmente, predisposição genética baseada em polimorfismos relacionados às drogas ou ao metabolismo ósseo tem sido apontada no desenvolvimento desta alteração óssea levando a um modelo multifatorial para ONMAB. ${ }^{29}$

Recentemente, pesquisas envolvendo a ação dos bisfosfonatos em células epiteliais mostram que estes medicamentos interferem na proliferação do epitélio de revestimento indicando que o efeito dos medicamentos sobre o tecido epitelial pode induzir a alteração de reparo tecidual e fazer parte da fisiopatologia da ONMAB. ${ }^{30}$

\section{Fatores de risco}

Vários fatores de risco para o desenvolvimento de ONMAB podem ser agrupados em fatores relacionados aos medicamentos, fatores locais, demográficos ou sistêmi$\cos ^{18,24,28,29,31}$

Os fatores de risco relacionados com o medicamento incluem a potência particular de cada bisfosfonato, por exemplo, o zoledronato é mais potente que o pamidronato, que é mais potente que os bisfosfonatos orais. A administração endovenosa parece conferir um risco mais alto do que a administração oral. A duração da terapia é importante, sendo que, quanto mais longa maior o risco para o desenvolvimento de osteonecrose. ${ }^{26}$

Bamias et $a l .{ }^{31}$ observaram que a incidência de ONMAB aumenta em 1,5\% entre pacientes tratados por 4-12 meses e em 7,7\% nos pacientes tratados com BFs durante 37 a 48 meses. A média de ocorrência de ONMAB nos pacientes em tratamento com ácido zoledrônico é de 16 meses, nos tratados com pamidronato é de 34 meses e com BFs orais de 54 meses.

Outros fatores de risco associados são o tipo de câncer, diagnóstico concomitante de osteopenia ou osteoporose durante o diagnóstico do câncer, terapia com corticóides e outros quimioterápicos, diabetes, uso de álcool e tabaco. ${ }^{18,26}$

Dentre os fatores locais foram descritos principalmente traumas cirúrgicos, como extração dentária ( $86 \%$ dos casos), pobre higiene oral, doenças infecciosas orais e trauma ocasionado por próteses removíveis. . $^{1820,23}$
Mavrokokki et al. ${ }^{32}$ observaram que $72 \%$ das ONMAB ocorrem em pacientes com doenças ósseas malignas. Nos casos detectados neste mesmo estudo, em $73 \%$ a ONMAB estava associada a extração dentária.

Apesar de a maioria dos casos de ONMAB estar associada a um fator desencadeante, alguns casos de surgimento espontâneo são relatados na literatura. ${ }^{17,19,20}$

\section{Aspectos imagenológicos}

Alterações radiográficas não são evidentes até que se tenha um extenso envolvimento ósseo. De qualquer forma, radiografias periapicais e panorâmicas podem não revelar significantes modificações em estágios iniciais da ON. Alterações tardias podem mimetizar patologias clássicas periapicais como espessamento do ligamento periodontal, osteomielites ou doença óssea primária (mieloma) ou metastática. Após longo período de tempo de uso dos BFs, pode ser observada osteoesclerose óssea, especialmente na lâmina dura. Tomografias computadorizadas auxiliam no diagnóstico e planejamento cirúrgico dos casos de ONMAB..$^{15,16-19,28}$

\section{Conduta terapêutica}

O tratamento da ONMAB é bastante variado, controverso e desafiador, visto que nenhum tratamento efetivo tem sido proposto até o momento. O protocolo é direcionado para cada caso dependendo do grau clínico da doença. $\mathrm{O}$ tratamento é integrado e envolve o uso de antibióticos, irrigação local com solução antimicrobiana, debridamento local da ferida, sequestrectomia, cirúrgica, uso de plasma rico em plaquetas e oxigenação hiperbárica. ${ }^{15,24,26,34}$

Pacientes com exposições ósseas assintomáticas podem ser tratados com irrigação com soluções antimicrobianas, como a clorexidina, rigoroso controle clínico e radiográfico, e uso de antibióticos sistêmicos, como penicilina ou clindamicina. Em alguns casos tem sido utilizado antibiótico sistêmico para prevenir infecção secundária. ${ }^{10,15,29,30}$ Nos casos em que há sequestro ósseo sintomático indica-se a remoção do osso necrótico, com menor agressão tecidual possível tanto ao osso como para o tecido mole adjacente. A irrigação constante com solução antimicrobiana e manutenção de antibioticoterapia por via oral pode ser o tratamento de escolha até o momento. ${ }^{15,35}$ Tratamento cirúrgico agressivo foi na maior parte dos casos ineficiente e frequentemente exacerbou os quadros de exposição óssea. ${ }^{18}$

Pacientes com drenagem em região sinusal e extensas áreas de exposição óssea ou grandes sequestros podem necessitar de procedimentos cirúrgicos mais extensos. Nos casos com drenagem de secreção purulenta, cultura e antibiograma devem ser realizados. ${ }^{20,22,26}$ Para muitos pacientes, a cicatrização completa pode nunca acontecer e eles terão que se resignar a viver com algum grau de exposição óssea. 
O tratamento pode limitar-se a promover analgesia e controlar a progressão da doença. ${ }^{15,36,37}$

Estudos de casos clínicos têm demonstrado a eficácia do uso de mediadores celulares, como o plasma rico em plaquetas (PRP) associado a debridamento cirúrgico, antibioticoterapia prolongada, irrigação com antimicrobianos e controle clínico e radiográfico para o tratamento da osteonecrose avascular dos maxilares. ${ }^{38}$

De acordo com Curi et al.,${ }^{38}$ o PRP é um concentrado autólogo de plaquetas humanas e uma fonte de variados fatores de crescimento que podem melhorar a cicatrização dos tecidos e a reparação óssea. Estes autores utilizaram o PRP em conjunto com medidas de debridamento cirúrgico, irrigação local e antibioticoterapia em três casos de ONMAB com o intuito de envolver os efeitos mecânicos da ressecção do osso necrótico combinado aos efeitos biológicos de osteoindução do PRP. Os autores concluíram que esse protocolo de tratamento mostrou resultados bastante satisfatórios, sendo que os casos tratados evoluíram com cicatrização e cura da osteonecrose. Outra vantagem observada em relação ao tratamento conservador foi a maior rapidez de resolução do problema e, com isso, uma melhor qualidade de vida aos pacientes.

A interrupção do tratamento com BFs tem sido considerada em casos graves; entretanto, deve-se considerar se os benefícios serão maiores do que o risco de eventos esqueléticos resultantes da interrupção do medicamento.

$\mathrm{Na}$ maior parte dos pacientes oncológicos, a manutenção do tratamento é primordial para a sobrevida do paciente. A interrupção no tratamento com BFs não assegura a melhora do quadro já instalado de ONMAB porque esta droga pode persistir por vários anos no tecido. ${ }^{19,24}$

O uso de oxigenação hiperbárica foi utilizada e inicialmente não mostrou resultados efetivos no tratamento da ONMAB, entretanto, novas evidências mostram alguns resultados promissores. ${ }^{35,39}$

\section{Conduta preventiva}

Marx et al. ${ }^{28}$ têm sugerido um protocolo de tratamento para os pacientes que necessitam realizar procedimentos cirúrgicos bucais e fazem uso de BFs. Neste protocolo levase em consideração o tipo e duração da terapia com bisfosfonatos, a descontinuidade com bisfosfonatos e o monitoramento do CTx (C-telopeptide) no soro ou na urina no momento da consulta e após a cirurgia. Neste estudo, os pacientes são divididos em três categorias: em uso de BFs há mais de três anos; em uso de BFs há menos de três anos sem sinais clínicos ou radiográficos de fator de risco para ONMAB e em uso de BFs há menos de três anos com um ou mais fatores de risco detectados clinica ou radiograficamente.

Uma vez que a terapia com bisfosfonatos tenha sido iniciada, uma rigorosa saúde oral deve ser mantida e todos os pacientes devem ser educados sobre a sua importância e a necessidade de um monitoramento clínico frequente pelo dentista.

O protocolo preventivo deve incluir: 1)avaliação odontológica (exame clínico e radiográfico) antes ou logo após iniciar o tratamento com os BFs. 2) remoção de focos de infecção e fatores traumáticos para a mucosa oral antes de iniciar o tratamento com BFs; 3) rígido controle de higiene oral para evitar infecções e complicações dentárias que possam ocasionar a osteonecrose; 4) esclarecimento do paciente quanto aos fatores de risco para o desenvolvimento da osteonecrose; 5) consultas frequentes ao cirurgião-dentista para avaliação das condições orais, controle de higiene, aplicação de flúor, monitoramento radiográfico, adaptação de próteses ( a cada seis meses); 6) quando for necessário procedimento invasivo na boca, o caso deve ser discutido entre o oncologista e o cirurgião-dentista; 7) monitoramento do tecido ósseo através do nível de CTx.

\section{Discussão}

Os BFs têm sido, indiscutivelmente, associados a melhora significativa da qualidade de vida dos pacientes portadores de metástases ósseas e com osteoporose grave. Sendo assim, vêm sendo utilizados em larga escala mundialmente e se estima que mais de três milhões de pacientes com câncer no mundo tenham recebido tratamento com BFs intravenosos desde sua introdução, além de um grande número de pacientes que recebem bisfosfonatos orais como alendronato (Fosamax $\left.{ }^{\circledR}\right)$ e risedronato (Actonel $\left.{ }^{\circledR}\right)$ para o tratamento de osteoporose pós-menopausa e induzida por glicocorticóides. ${ }^{15}$

A ONMAB é uma importante complicação oral associada ao uso dos BFs que, apesar de ter sido descrita recentemente, já mostra vários casos relatados na literatura. Esta necrose óssea tem mostrado um comportamento indolente, de difícil controle, podendo levar a exposição óssea crônica e quadros infecciosos persistentes. Desta forma, os cirurgiões-dentistas e médicos devem estar familiarizados com esta alteração e ter atenção especial no tratamento de todos os pacientes que fazem uso crônico dos BFs, tendo em vista que os mesmos alteram o remodelamento e reparo ósseo. $^{22}$

Apesar de vários trabalhos mostrarem uma baixa incidência da ONMAB, acredita-se que estes números poderão aumentar, tendo em vista que um número cada vez maior de pessoas faz uso destes medicamentos e que o efeito dos BFs é cumulativo e persistente no tecido ósseo. Até o presente momento, o maior número de casos relatados é em pacientes que fazem uso de BFs endovenosos, em protocolos de tratamento oncológico. Isso ocorre porque a potência relativa dos BFs usados é maior, entretanto, acredita-se que à medida que os pacientes utilizam os BFs orais por um longo período de tempo passarão a ter um risco maior de desenvolver 
ONMAB. De acordo com Lin et al., ${ }^{40}$ os BFs podem permanecer no osso por 12 anos após a terapia ter sido descontinuada. Essa propriedade dos BFs faz com que a ONMAB possa ocorrer mesmo após a interrupção do medicamento. Portanto, pacientes que fazem ou fizeram uso de BFs devem participar de um rigoroso protocolo de prevenção de ONMAB.

O tratamento da ONMAB é bastante complexo e diversos protocolos terapêuticos vêm sendo descritos na literatura com índices variáveis de sucesso. Assim, pensando nesta forma de osteonecrose, a prevenção é fundamental, pois a maior parte dos casos é desencadeada por algum fator traumático, quadros infecciosos que levam ao rompimento da mucosa oral, infecção, exposição e necrose óssea. O protocolo preventivo deve incluir avaliação clínica e imagenológica oral antes do tratamento com BFs ser iniciado, para eliminar focos de infecção, seguido de consultas odontológicas periódicas para eliminar possíveis fatores traumáticos, monitoramento do nível do metabolismo ósseo pelo CTx e orientação dos pacientes quanto aos riscos de desenvolverem a ONMAB.

Apesar da incidência e a patogênese da ONMAB ainda não estarem totalmente definidas, sabe-se que os BFs estão relacionados a uma emergente entidade clínica de comportamento indolente e difícil tratamento. $\mathrm{Na}$ anamnese realizada durante o tratamento odontológico, o cirurgião-dentista deve estar alerta pra identificar pacientes usuários crônicos de BFs e poder prevenir as complicações decorrentes do uso desta droga. Os oncologistas, por outro lado, devem solicitar aos pacientes que façam avaliação odontológica prévia e mantenham saúde oral.

A maior parte dos estudos publicados são relatos de casos ou de série de casos, de forma retrospectiva, o que gera várias limitações na obtenção de informações nos trabalhos. Medidas terapêuticas e preventivas mais eficazes poderão ser analisadas de forma mais aprimorada através de estudos prospectivos.

\footnotetext{
Abstract

Bisphosphonates are drugs used in the treatment of malignant metastatic diseases and in other bone lesions such as osteoporosis and Paget's disease. Besides their benefits, jaw osteonecrosis, an important side effect, has been observed in long-term users of these drugs. Jaw osteonecrosis is clinically characterized by prolonged maxillary and mandible bone exposure accompanied by osteomyelitis. These lesions are usually symptomatic and difficult to treat. This study has the objective of reviewing publications from 2003 to 2008 about bisphosphonate-associated jaw osteonecrosis, in particular in relation to oncology. Risk factors, and etiopathological, clinical, radiographic, therapeutic, and preventive aspects of this condition are presented and discussed. Due to the difficulty to treat this disease, the focus must be prevention, with the ideal therapy being the elimination of oral infections before treatment with bisphosphonates is initiated thereby attempting to
}

minimize possible traumas to the mouth with the use of these medications. Rev. Bras. Hematol. Hemoter. 2009; 31(1):41-46.

Key words: Review; physiopathology; osteonecrosis; diagnosis; therapy; oral manifestations.

\section{Referências Bibliográficas}

1. Hortobagyi GN, Theriault RL, Lipton A, Porter L, Blayney D, Sinoff C, et al. Long-term prevention of skeletal complications of metastatic breast cancer with pamidronate. Protocol 19 Aredia Breast Cancer Study Group. J Clin Oncol. 1998;16(6):2038-44.

2. Conte PF, Giannessi PG, Latreille J, Mauriac L, Koliren L, Calabresi $\mathrm{F}$, et al. Delayed progression of bone metastases with pamidronate therapy in breast cancer patients: a randomized, multicenter phase III trial. Ann Oncol. 1994;5 Suppl 7:S41-4.

3. Hillner BE, Ingle JN, Berenson JR, Janjan NA, Albain KS, Lipton A, et al. American Society of Clinical Oncology guideline on the role of bisphosphonates in breast cancer. American Society of Clinical Oncology Bisphosphonates Expert Panel. J Clin Oncol. 2000;18(6):1378-91.

4. Berenson JR, Hillner BE, Kyle RA, Anderson K, Lipton A, et al. American Society of Clinical Oncology clinical practice guidelines: the role of bisphosphonates in multiple myeloma. J Clin Oncol. 2002;20(17):3719-36.

5. van Holten-Verzantvoort AT, Kroon HM, Bijvoet OL, Cleton FJ, Beex LV, Blijham G, et al. Palliative pamidronate treatment in patients with bone metastases from breast cancer. J Clin Oncol. 1993;11(3):491-8.

6. Berenson JR, Lichtenstein A, Porter L, Dimopoulos MA, Bordoni $\mathrm{R}$, George $\mathrm{S}$, et al. Long-term pamidronate treatment of advanced multiple myeloma patients reduces skeletal events. Myeloma Aredia Study Group. J Clin Oncol. 1998;16(2):593-602.

7. Hortobagyi GN, Theriault RL, Porter L, Blayney D, Lipton A, Sinoff $\mathrm{C}$, et al. Efficacy of pamidronate in reducing skeletal complications in patients with breast cancer and lytic bone metastases. N Engl J Med. 1996;335(24):1785-91.

8. Berenson JR, Lichtenstein A, Porter L, Dimopoulos MA, Bordoni $\mathrm{R}$, George S, et al. Efficacy of pamidronate in reducing skeletal events in patients with advanced multiple myeloma. N Engl J Med. 1996;334(8):488-93.

9. Theriault RL, Lipton A, Hortobagyi GN, Leff R, Glück S, Stewart $\mathrm{JF}$, et al. Pamidronate reduces skeletal morbidity in women with advanced breast cancer and lytic bone lesions: a randomized, placebo-controlled trial. Protocol 18 Aredia Breast Cancer Study Group. J Clin Oncol. 1999;17(3):846-54.

10. Rodan GA, Fleisch HA. Bisphosphonates: mechanisms of action. J Clin Invest. 1996;97(12):2692-6.

11. Hughes DE, MacDonald BR, Russell RG, Gowen M. Inhibition of osteoclast-like cell formation by bisphosphonates in long-term cultures of human bone marrow. J Clin Invest. 1989;83(6):1930-5.

12. Hughes DE, Wright KR, Uy HL, Sasaki A, Yoneda T, Roodman $\mathrm{GD}$, et al. Bisphosphonates promote apoptosis in murine osteoclasts in vitro and in vivo. J Bone Miner Res. 1995;10(10):1478-87.

13. Murakami H, Takahashi N, Sasaki T, Udagawa N, Tanaka S, Nakamura I, et al. A possible mechanism of the specific action of bisphosphonates on osteoclasts: tiludronate preferentially affects polarized osteoclasts having ruffled borders. Bone. 1995;17(2): $137-44$

14. Sahni M, Guenther HL, Fleisch H, Collin P, Martin TJ. Bisphosphonates act on rat bone resorption through the mediation of osteoblasts. J Clin Invest. 1993;91(5):2004-11. 
15. Ruggiero SL, Gralow J, Marx RE, Hoff AO, Schubert MM, Huryn JM, et al. Practical guidelines for the prevention, diagnosis and treatment of osteonecrosis of the jaw in patients with cancer. $J$ Clin Oncol Prac 2006;2:7-14.

16. Migliorati CA. Bisphosphanates and oral cavity avascular bone necrosis. J Clin Oncol. 2003;21(22):4253-4

17. Migliorati CA. Bisphosphonate-associated oral osteonecrosis. Oral Surg Oral Med Oral Pathol Oral Radiol Endod. 2005; 99 (2): 135

18. Migliorati CA, Casiglia J, Epstein J, Jacobsen PL, Siegel MA, Woo SB. Managing the care of patients with bisphosphonate-associated osteonecrosis: an American Academy of Oral Medicine position paper. J Am Dent Assoc. 2005;136(12):1658-68.

19. Migliorati CA, Schubert MM, Peterson DE, Seneda LM. Bisphosphonate-associated osteonecrosis of mandibular and maxillary bone: an emerging oral complication of supportive cancer therapy. Cancer. 2005;104(1):83-93.

20. Marx RE, Sawatari Y, Fortin M, Broumand V. Bisphosphonateinduced exposed bone (osteonecrosis/osteopetrosis) of the jaws: risk factors, recognition, prevention, and treatment. J Oral Maxillofac Surg. 2005;63(11):1567-75.

21. Woo SB, Hellstein JW, Kalmar JR. Narrative [corrected] review: bisphosphonates and osteonecrosis of the jaws. Ann Intern Med. 2006;144(10):753-61.

22. Lam DK, Sándor GK, Holmes HI, Evans AW, Clokie CM. A review of bisphosphonate-associated osteonecrosis of the jaws and its management. J Can Dent Assoc. 2007;73(5):417-22.

23. Durie BG, Katz M, Crowley J.Osteonecrosis of the jaw and bisphosphonates. N Engl J Med. 2005;353(1):99-102.

24. Advisory Task Force on Bisphosphonate-Related Ostenonecrosis of the Jaws, American Association of Oral and Maxillofacial Surgeons. American Association of Oral and Maxillofacial Surgeons position paper on bisphosphonate-related osteonecrosis of the jaws. J Oral Maxillofac Surg. 2007;65(3):369-76.

25. Hess LM, Jeter JM, Benham-Hutchins M, Alberts DS. Factors associated with osteonecrosis of the jaw among bisphosphonate users. Am J Med. 2008;121(6):475-83.e3.

26. Ruggiero SL, Mehrotra B, Rosenberg TJ, Engroff SL. Osteonecrosis of the jaws associated with the use of bisphosphonates: a review of 63 cases. J Oral Maxillofac Surg. 2004;62(5):527-34.

27. Schwartz HC. Osteonecrosis and bisphosphonates: correlation versus causation. J Oral Maxillofac Surg. 2004;62(6):763-4.

28. Marx RE, Sawatari Y, Fortin M, Broumand V. Bisphosphonateinduced exposed bone (osteonecrosis/osteopetrosis) of the jaws: risk factors, recognition, prevention, and treatment. J Oral Maxillofac Surg. 2005;63(11):1567-75.

29. Hewitt C, Farah CS. Bisphosphonate-related osteonecrosis of the jaws: a comprehensive review. J Oral Pathol Med. 2007; 36 (6):319-28

30. Landesberg R, Cozin M, Cremers S, Woo V, Kousteni S, Sinha S, et al. Inhibition of oral mucosal cell wound healing by bisphosphonates. J Oral Maxillofac Surg. 2008;66(5):839-47.

31. Marx RE. Oral and intravenous bisphosphonate-induced osteonecrosis of the jaws. History, etiology, prevention, and treatment. Quintessence Publishing CO Inc; 2007. p.9-96.

32. Bamias A, Kastritis E, Bamia C, Moulopoulos LA, Melakopoulos I, Bozas G, et al.Osteonecrosis of the jaw in cancer after treatment with bisphosphonates: incidence and risk factors. J Clin Oncol. 2005;23(34):8580-7.

33. Mavrokokki T, Cheng A, Stein B, Goss A. Nature and frequency of bisphosphonate-associated osteonecrosis of the jaws in Australia. J Oral Maxillofac Surg. 2007;65(3):415-23.
34. Engroff SL, Kim DD. Treating bisphosphonate osteonecrosis of the jaws: is there a role for resection and vascularized reconstruction? J Oral Maxillofac Surg. 2007;65(11):2374-85.

35. Biasotto M, Chiandussi S, Dore F, Rinaldi A, Rizzardi C, Cavalli F, et al. Clinical aspects and management of bisphosphonatesassociated osteonecrosis of the jaws. Acta Odontol Scand. 2006; 64(6):348-54.

36. Ruggiero S, Woo V. Mehrotra B, Fantasia J. Osteonecrosis of the jaws associated with the use of bisphosphonate medications: a report of 60 cases. Oral Surg Oral Med Oral Pathol Oral Radiol Endod 2004;98:196.

37. Diego R, D'Orto O, Pagani D, Agazzi A, Marzano U, Derada Troletti G, et al. Bisphosphonate-associated osteonecrosis of the jaws: a therapeutic dilemma. Oral Surg Oral Med Oral Pathol Oral Radiol Endod. 2007;103(3):e1-5.

38. Curi MM, Cossolin GS, Koga DH, Araújo SR, Feher O, dos Santos MO, et al. Treatment of avascular osteonecrosis of the mandible in cancer patients with a history of bisphosphonate therapy by combining bone resection and autologous platelet-rich plasma: Report of 3 cases. J Oral Maxillofac Surg. 2007;65(2):349-55.

39. Shimura K, Shimazaki C, Taniguchi K, Akamatsu S, Okamoto M, Uchida $\mathrm{R}$, et al. Hyperbaric oxygen in addition to antibiotic therapy is effective for bisphosphonate-induced osteonecrosis of the jaw in a patient with multiple myeloma. Int $\mathrm{J}$ Hematol. 2006;84(4):343-5.

40. Lin JH, Russell G, Gertz B. Pharmacokinetics of alendronate: an overview. Int J Clin Pract Suppl. 1999;101:18-26.

Avaliação: Editor e dois revisores externos

Conflito de interesse: não declarado

Recebido: 06/05/2008

Aceito após modificações: 05/06/2008 